Sriwijaya Journal of Ophthalmology(SJO) http://sriwijayaopthalmology.com

Vol 2 Issue 22019

\title{
Diagnosis and Management of Bleeding Vitreous by Trauma Closed Eyeball
}

\author{
Rika Anggraini ${ }^{1 *}$, AK Ansyori ${ }^{1}$, Ramzi Amin $^{1}$ \\ ${ }^{1}$ Department of Ophthalmology, Faculty of Medicine, Universitas Sriwijaya, Indonesia \\ *Correspondence author email: rika.anggraini@gmail.com
}

\begin{abstract}
Introduction. Diagnosis of vitreous bleeding is generally straightforward and it can be made through history, ophthalmological examination and fundoscopy. However, the management is determined by the underlying etiology. The most common causes as factors that cause opposite vitreous bleeding include retinopathy of proliferative diabetes, posterior vitreous detachment (PVD) and trauma in 59-88.5\% of cases. Reported a case of vitreous hemorrhage due to closed eyeball trauma performed by pars plana vitrectomy and endolaser with significant vision improvement.
\end{abstract}

Case Presentation. A male, 21 years old, student, lived in suburban, came to the Polyclinic of RSMH on July 2019. Approximately 1 month ago the patient complained that his right eye vision suddenly felt blurry after being hit by volleyball from the front from a distance of 1 meter while playing volleyball with his friends. Patients complain of the view of the right eye as if it were covered with a red shadow accompanied by pain around the right eye. Ophthalmological examination obtained vision of this patient is a wave of the hand that is $1 / 300$ in the right eye and 6/6 in the left eye that shows the disorder is unilateral with a fairly poor decrease in vision. During intraoperative right eye hemorrhage was found to nearly cover the retina with the source of bleeding in peripheral retinal blood vessels which when found intraoperatively were not found extravasation or active bleeding.

Conclusion. The operative action of pars plana vitrectomy gives a clinically significant result that is obtained very good sharp eyesight and from the examination results obtained echofree on vitreous. Proper timing is very important in determining the management of vitreous bleeding in order to get good results and minimize the risk of complications that can occur.

Keywords. bleeding vitreous, trauma closed eye ball, management, diagnosis 


\section{Introduction}

The sudden and progressive sharp decrease in vision due to vitreous bleeding is one of the emergency conditions and can become irreversible if not treated. The main problem that must be considered is the etiological factor that causes the onset of blood in the vitreous and the sharp decrease in vision that accompanies it. The incidence of vitreous bleeding occurs 7 : 100,000 cases, which makes it one of the most common causes of visual impairment from acute or subacute. Although the diagnosis of vitreous bleeding is generally straightforward, it can be made through history, ophthalmological examination and fundoscopy, but management is determined by the underlying etiology. ${ }^{1}$

The diagnosis of vitreous hemorrhage itself is nonspecific and the cause is very broad. Goff, et al. divides the various etiologies of vitreous bleeding into etiology of blood vessels, anterior segments, vitreoretina, tumors, trauma, inflammation and blood disorders. The most common causes as factors that cause opposite vitreous bleeding include retinopathy of proliferative diabetes, posterior vitreous detachment (PVD) and trauma in 59-88.5\% of cases. ${ }^{2}$

In old age, the most common cause of vitreous bleeding is diabetic retinopathy, especially proliferative type, which is approximately $60 \%$, while in young age, the most common cause is trauma, which is approximately $68.5 \%$, which most often causes a sharp decrease in irreversible monocular vision. ${ }^{3,4}$ At a young age, vitreous bleeding due to trauma can be caused by either closed trauma or blunt trauma or open trauma or penetrating trauma. In closed trauma or blunt trauma must be aware of the retinal tear that accompanies vitreous bleeding which must be treated immediately. While cases of vitreous bleeding in cases of open trauma are usually accompanied by damage to other ocular parts such as the rupture of the sclera, iris, ciliary body to the central retina. ${ }^{1,4}$

In general, if vitreous hemorrhage is not managed properly and quickly or treated conservatively, it is believed to pose a risk of complications such as retinal detachment, glaucoma and pigmentary retinopathy. ${ }^{3,4}$ vitrectomy, so that cases of vitreous bleeding at a young age due to trauma without any complications if handled quickly and appropriately can provide a good and reversible prognosis.

Reported a case of vitreous hemorrhage due to closed eyeball trauma performed by pars plana vitrectomy and endolaser with significant vision improvement.

\section{Case Report}


A male, 21 years old, student occupation, address outside the city, came to the Polyclinic of RSMH on July 2, 2019 with medical records 112241. Anamnesis (Auto-anamnesis) Main Complaints: Right eye vision blurred since, 1 month ago. Travel History of the Disease: Since, 1 month ago the patient complained of vision in the opaque right eye, blurred felt suddenly after being hit by volleyball from the front with a distance of \pm 1 meter when playing volleyball with his friends. Sufferers complain that their eyes are covered in red shadows. Pain (+) around the eyes, bleeding from the right eye (-), discharge such as egg whites from the eyeball (-). Complaints of red eyes (-), glare (-), vision like a curtain (-), vision like a rainbow (-). Patients treated at hospitals in the area but there was no change. Since, 1 week ago the patient's right eye view became blurry. Pain (-), red eyes (-). Patients treated at Myria Hospital were then referred to RSMH.

Past history of disease History of diabetes is denied, history of high blood pressure is denied, history of wearing sunglasses is denied, history of coughing, straining and lifting heavy objects is denied, history of the same disease in the family is denied.

Physical examination of generalist status is General condition: Good, Awareness: Compost mentis, Blood Pressure: 110/80 mmHg, Pulse: 82 x / minute, Respiration: 20 x / minute, Temperature: Afebris.

Ophthalmologic Status Table (2 July 2019):

\begin{tabular}{|c|c|c|}
\hline & OD & OS \\
\hline Vision & $1 / 300$ & $6 / 6$ \\
\hline TIO & $17,3 \mathrm{mmHg}$ & $15,6 \mathrm{mmHg}$ \\
\hline KBM & \multicolumn{2}{|c|}{ Ortoforia } \\
\hline GBM & Good in all directions & Good in all directions \\
\hline Palpebra & Quite & Quite \\
\hline Conjunctiva & Quite & Quite \\
\hline Cornea & Clear & Clear \\
\hline BMD & Medium & Medium \\
\hline Iris & Good image & Good image \\
\hline Pupil & Round, central, RC (+), Ø $3 \mathrm{~mm}$ & Round, central, RC (+), Ø $3 \mathrm{~mm}$ \\
\hline Lens & Clear & Clear \\
\hline
\end{tabular}




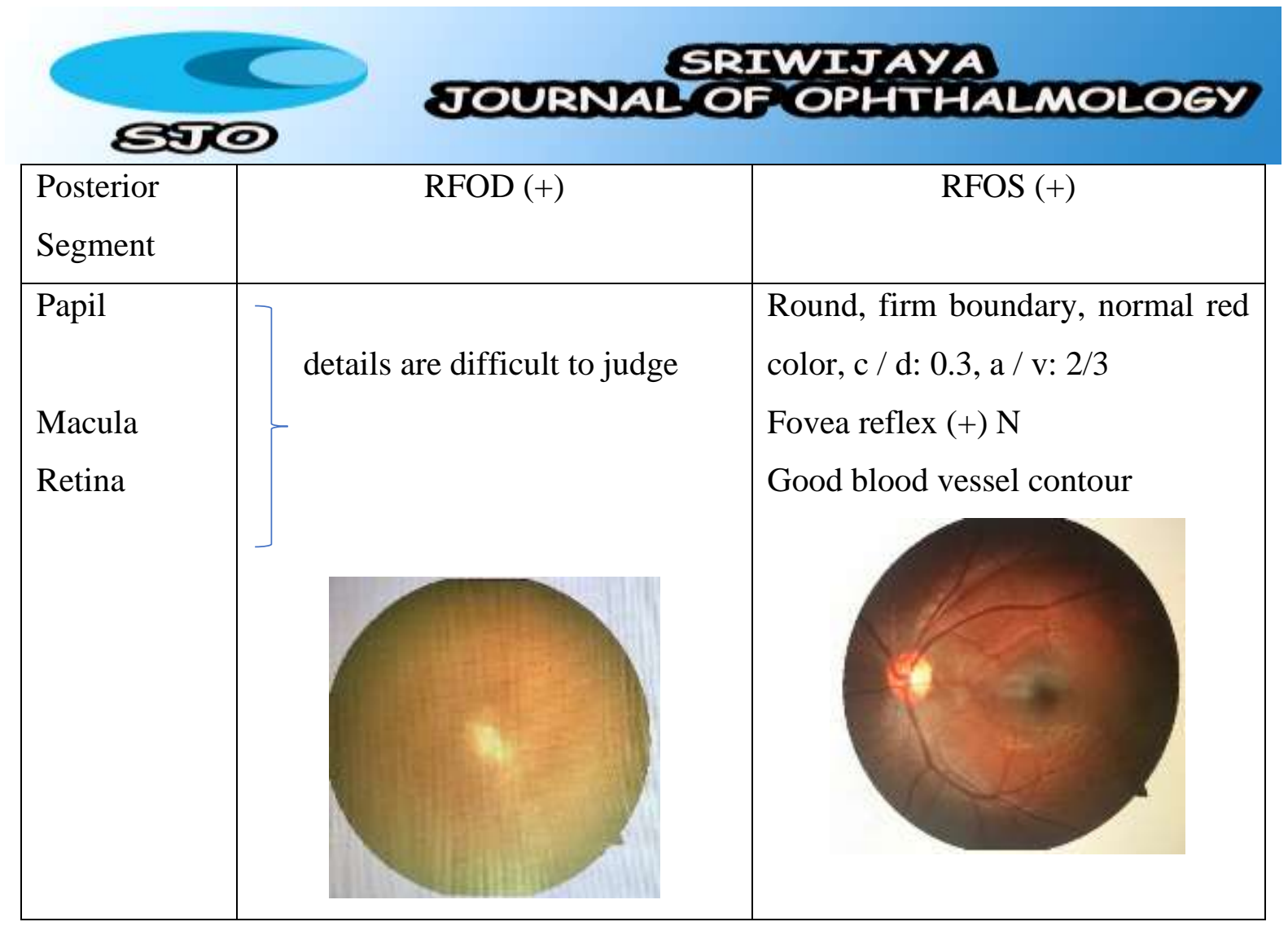

Image of right-eye orbital ultrasound (2 July 2019)

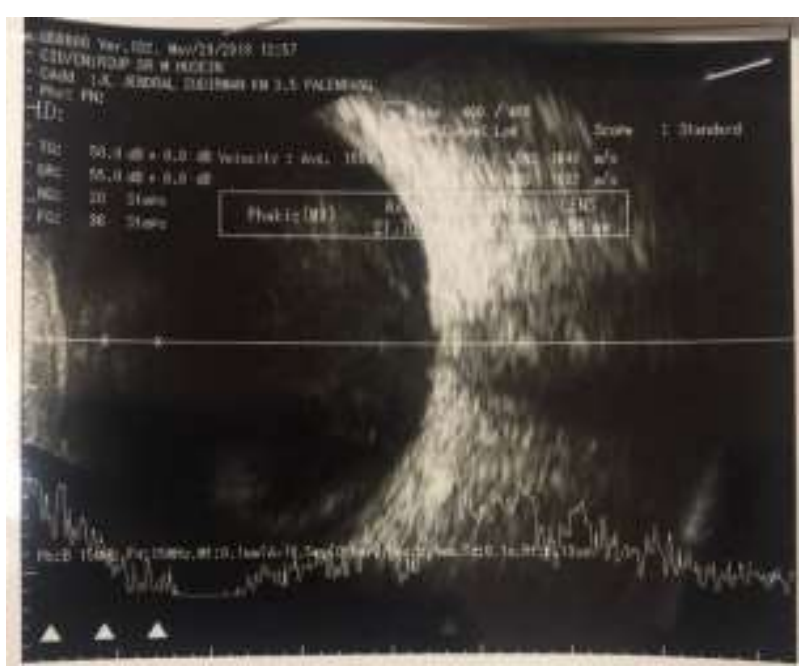

Retina: intact, Vitreus: echospike, high reflectivity, high mobility, Choroid: not thickened, Axial length: $23.04 \mathrm{~mm}$. Impression: vitreous bleeding

The differential diagnosis is vitreous hemorrhage OD ec dextra closed eyeball trauma, vitreous hemorrhage ec ecales disease, vitreous hemorrhage OD ec valsalva maneuver. 
Diagnosis of vitreous bleeding OD ec closed trauma to the eyeball dextra. The treatment is informed consent, Pro pars plana vitrectomy + OD endolaser, Pro laboratory check, Pro X-ray chest X, Pro Consultant Anesthesia. Prognosis of Quo ad vitam: Bonam, Quo ad functionam: Dubia ad bonam.

\section{Discussion}

History taking, history of the disease and ophthalmological examination and investigations are very important to explore information about the onset, causes and predisposing factors of a case of vitreous bleeding. All this information can help in establishing the diagnosis and planning a management. A man, 21 years old, with right eye complaints, has suddenly reported blurring \pm 1 month ago.

Approximately 1 month ago the patient complained that his right eye vision suddenly felt blurry after being hit by volleyball from the front from a distance of 1 meter while playing volleyball with his friends. Patients complain of the view of the right eye as if it were covered with a red shadow accompanied by pain around the right eye. Patients do not seek treatment until 1 week ago the patient's right eye view became blurred without red eyes and without pain. From the history it was found that patient complaints tend to lead to symptoms of sudden vision loss without pain and without red eyes. Patients' complaints about views such as being covered in red shadows can be caused by the presence of blood in the visual axis of both the posterior and posterior segments.

The history in this patient is strong in explaining the cause of the complaint, namely trauma. The most etiology of vitreous bleeding at age $<40$ years is trauma either penetrating trauma, or closed trauma that is equal to $67 \%$. Patients are also not accompanied by a history of previous illnesses such as hypertension, diabetes mellitus, blood disorders, tuberculosis so that systemic causes that can cause vitreous bleeding such as vascular etiology and blood disorders can be ruled out.

Ophthalmological examination obtained vision of this patient is a wave of the hand that is $1 / 300$ in the right eye and $6 / 6$ in the left eye that shows the disorder is unilateral with a fairly poor decrease in vision. From the anterior segment examination both eyes were found to be calm and within normal limits. Examination of the posterior segment using fundus-copy in the patient's right eye is difficult to assess, so an ultrasound B-scan was performed and a high reflectivity and mobility of vitreous echospike was obtained which described the presence of fluid which was still classified as subacute, with an intact retina. So, from the above 
examination it can be concluded in patients with vitreous bleeding in the right eye that is most likely due to trauma suffered by the patient 1 month ago.

The differential diagnosis of this patient based on age can be diagnosed with Eales disease and valsava maneuver, both of which occur in the second decade of age. But the diagnosis of Eales disease can be ruled out because the patient has no history of tuberculosis, is not accompanied by signs of inflammation, ischemic, neovascularization of the retinal blood vessels and the occurrence of vitreous hemorrhage is unprecedented in which Eales disease is characterized by inflammation, ischemic recurrent vitreous bleeding. The etiological diagnosis of valsalva maneuver can also be excluded because from a previous history there is no history of coughing, straining or lifting heavy loads that can increase intrathoraxic pressure and intrabdominal pressure.

During intraoperative right eye hemorrhage was found to nearly cover the retina with the source of bleeding in peripheral retinal blood vessels which when found intraoperatively were not found extravasation or active bleeding. In vitreous bleeding due to trauma, the most frequently involved are the branches of the retinal vein arteries and the posterior ciliary arteries. So that after vitrectomy surgery is performed, an endo-laser is performed on peripheral blood vessels to prevent rebleeding.

Ultrasound examination (USG) is very helpful to assess the state of the posterior segment with vitreous that is not clear. Ultrasound can be seen from the echospike which indicates vitreous clarity. With high reflectivity which is usually present in liquids, it can be concluded in patients with vitreous bleeding. The retina is intact without any detachment, although sometimes the tear retina is difficult to identify from ultrasound. After vitrectomy and endo-laser surgery in patients, there was a very significant visual improvement from 1/300 to 6/6. Proper timing in determining the management of vitreous bleeding can improve vision, improve the source of bleeding, shorten the time of vision loss and reduce the risk of more severe complications. Vitrectomy itself was chosen because in patients with onset of hemorrhage already 1 month, which means that within 1 month there was no spontaneous reabsorption but there were complaints of increasingly blurred vision where vitrectomy was indicated one of them in cases of vitreous bleeding that did not disappear after observation.

The prognosis in this patient quo ad functionam is dubia ad bonam because in vitreous hemorrhage cases with known etiology are trauma without complications such as retinal detachment then proper handling of patients is expected to restore sharp eyesight for the better. Intraoperative complications must also be considered in determining the prognosis because in 
vitrectomy surgery there can be a risk of complications such as retinal detachment or choroidal bleeding which will worsen visual function.

\section{Conclusion}

Reports of cases of vitreous hemorrhage have been reported due to closed-eyed trauma in young patients undergoing pars plana and endo-laser operative vitrectomy. Basically, it can be observed if the onset is still expected to spontaneously reabsorb. In cases of non-diminished bleeding accompanied by a significant decrease in vision, operative measures are needed. The operative action of pars plana vitrectomy gives a clinically significant result that is obtained very good sharp eyesight and from the examination results obtained echofree on vitreous. Proper timing is very important in determining the management of vitreous bleeding in order to get good results and minimize the risk of complications that can occur.

\section{References}

1. "National Data." Vision Health Initiative (VHI). Centers for Disease Control and $\begin{array}{lllll}\text { Prevention. } & 30 & \text { Sept. } & 2015 . & \text { Available from }\end{array}$ https://www.cdc.gov/visionhealth/data/national.htm

2. American Academy of Ophthalmology. Diabetic Retinopathy, Preferred Practice Pattern. San Francisco: American Academy of Ophthalmology, 2017

3. Tan HS, Mura M and Bijl HM. Early vitrectomy for vitreous hemorrhage associated with retinal tears. Am J Ophthalmol 2010; 150: 529-533

4. P. Rishi, E. Rishi, A. Gupta, M. Swaminathan, and J. Chhablani, "Vitreous hemorrhage in children and adolescents in India," Journal of AAPOS, vol. 17, no. 1, pp. 64-69, 2013.

5. Retina and Vitreous. American Academy of Ophthalmology. Canada. 2017-2018.

6. Fundamentals and Principles of Ophthalmology. American Academy of Ophthalmology. Canada. 2017-2018.

7. La Goff MM, Bishop PN. Adult vitreous structure and postnatal changes. Eye. 2008;22:1214-22.

8. Tan HS, Mura M, Bijl HM. Early vitrectomy for vitreous hemorrhage associated with retinal tears. Am J Ophthalmol 2010; 150: 529-533 
9. Chuang LH, Wang NK, Chen YP, et al. Vitrectomy and panretinal photocoagulation reduces the occurrence of neovascular glaucoma in central retinal vein occlusion with vitreous hemorrhage. Retina2013; 33: 798-802

10. Zhang T, Zhang J, Sun X, et al. Early vitrectomy for dense vitreous hemorrhage in adults with non-traumatic and non-diabetic retinopathy. j Int Med Res. 2017 Dec; 45(6):2065-2071

11. Witmer MT, Cohen SM. Oral anticoagulation and the risk of vitreous hemorrhage and retinal tears in eyes with acute posterior vitreous detachment. Retina. 2013 Mar;33(3):621-6.

12. Chopdar, A. \& Aung, T. 2014. Multimodal retinal imaging. JP Medical Ltd.

13. Bhavsar AR, Torres K, Beck, RW, et al.; Diabetic Retinopathy Clinical Research Network Study Investigators. Randomized Clinical Trial Evaluating Intravitreal Ranibizumab or Saline for Vitreous Hemorrhage from Proliferative Diabetic Retinopathy. JAMA Ophthalmol. 2013 Mar; 131(3): 283-293.

14. Bond-Taylor, Martin, Jakobsson G, Zetterberg M. "Posterior vitreous detachmentprevalence of and risk factors for retinal tears." Clinical Ophthalmology (Auckland, NZ) 11 (2017): 1689.

15. Biswas J, Ravi RK, Naryanasamy A, et al. Eales' disease-current concepts in diagnosis and management. J Ophthalmic Inflamm Infect 2013; 3: 11-11.

16. Annan J, Carvounis P. Current Management of Vitreous Hemorrhage due to Proliferative Diabetic Retinopathy. Int Ophthalmol Clin. 2014 Spring; 54(2): 141-153 